\title{
Efeito do Sistema de Plantio e Doses do Nicosulfuron sobre a Atividade MicRobiana do Solo ${ }^{1}$
}

\author{
Effect of Planting System and Nicosulfuron Doses on Soil Microbial Activity \\ CAMELO, G.N. ${ }^{2}$, SANTOS, J.B. ${ }^{3}$, LAZARI, T.M. ${ }^{4}$, OLIVEIRA, T.A. ${ }^{5}$, SANTOS, E.A. ${ }^{6}$, \\ FERREIRA, E.A. ${ }^{7}$ e PEREIRA, G.A.M. ${ }^{8}$
}

\begin{abstract}
RESUMO - Objetivou-se com este trabalho avaliar o efeito do atrazine em mistura com nicosulfuron sobre a atividade microbiológica do solo em sistema de cultivo convencional ou semeadura direta. O estudo foi realizado em área de integração milho-braquiária, sendo feitas aplicações dos herbicidas atrazine + nicosulfuron em duas dosagens $(1.500+10$ e $1.500+30 \mathrm{~g} \mathrm{~h}^{-1}$ ) e mantidas duas áreas testemunhas: não capinada e capinada sem cultivo. Por ocasião do florescimento, foram retiradas amostras do solo em cada unidade experimental, para determinação da taxa de desprendimento de $\mathrm{CO}_{2}$, carbono da biomassa microbiana $(\mathrm{CBM})$ e quociente metabólico $\left(q \mathrm{CO}_{2}\right)$. Entre os sistemas de cultivo, a taxa de desprendimento de $\mathrm{CO}_{2}$ foi menor nas parcelas não capinadas. O CBM foi maior no sistema de plantio direto, sendo afetado negativamente pela ausência de vegetação. Menores valores para CBM foram observados nas amostras de solo das parcelas capinadas. Os maiores valores de $q \mathrm{CO}_{2}$ foram observados no sistema convencional. No sistema de plantio direto não se verificou diferença entre os tratamentos cujo solo recebeu herbicida e o isento de capinas. Dessa forma, podese concluir que os indicadores microbiológicos avaliados foram sensiveis aos tratamentos propostos, indicando menor distúrbio ao solo quando em sistema de semeadura direta. Entretanto, na integração lavoura-pecuária em sistema convencional de cultivo, o efeito negativo dos herbicidas é aumentado com o incremento na dose do nicosulfuron.
\end{abstract}

Palavras-chave: biomassa microbiana, respirometria, quociente metabólico.

\begin{abstract}
This study aimed to evaluate the effect of atrazine + nicosulfuron on soil microbial activity, under the conventional and no-tillage systems. The experiment was carried out in an area of maize-brachiaria intercropping, where atrazine + nicosulfuron were applied at $(1,500+10$ and 1,500 $+30 \mathrm{~g} \mathrm{~h}^{-1}$ ) with a weed-handed area being maintained without prior cultivation and another area without weed control. At flowering, soil samples were collected to determine the rate of $\mathrm{CO}_{2}$ unfastening, microbial biomass carbon $(\mathrm{MBC})$ and metabolic quotient $\left(q \mathrm{CO}_{2}\right)$ using the relation between accumulated $\mathrm{CO}_{2}$ and $M B C$. The rate of $\mathrm{CO}_{2}$ unfastening was lower only in plots without weed control. $M B C$ was higher in the no-tillage system, being negatively affected due to the absence of vegetation. Lower MBC was observed in the weed-handed areas, probably due to the absence of straw. Under no-tillage system conditions, no difference was observed between the soil treatments with herbicide application and those without weed control. It can be concluded that the evaluated microbiological indicators were sensitive to the treatments, showing lower soil disturbance under no-tillage system conditions. However, in crop-livestock integration under the conventional system, the negative herbicide effect increase of nicosulfuron rate.
\end{abstract}

Keywords: microbial biomass, respirometry, metabolic quotient.

1 Recebido para publicação em 3.5.2010 e aprovado em 25.6.2011.

2 Engo-Agr ${ }^{0}$, Doutorando em Fitotecnia, Universidade Federal de Viçosa - DFT/UFV, Campus Universitário, 36570-000 ViçosaMG,<gessimarcamelo@yahoo.com.br>; ${ }^{3}$ Eng - -Agr ${ }^{\circ}$, D.Sc., Professor do Departamento de Produção Vegetal da UFVJM, <jbarbosasantos@yahoo.com.br>; ${ }^{4}$ Mestrando em Agroenergia - Universidade Federal do Tocantins - UFT; ${ }^{5}$ M.Sc. em Entomologia - Instituto Nacional de Pesquisas da Amazônia - INPA; ${ }^{6}$ Doutorando em Produção Vegetal - Universidade Estadual Paulista Júlio de Mesquita Filho - UNESP, ${ }^{7}$ Engo-Agr ${ }^{0}$, D.Sc., Bolsista PNPD-CAPES do Departamento de Produção Vegetal da UFVJM, <evanderalves@yahoo.com.br>; ${ }^{8}$ Eng $^{-}$-Agr ${ }^{\circ}$, Mestrando do Departamento de Produção Vegetal da UFVJM.

Planta Daninha, Viçosa-MG, v. 29, n. 4, p. 829-835, 2011 


\section{INTRODUÇÃO}

De acordo com FEBRAPDP (2008), o uso do sistema de plantio direto (SPD) trouxe muitas mudanças ambientais positivas, como a redução significativa dos níveis de contaminação dos cursos das águas, a maior estabilidade ecológica para as lavouras, a alteração da flora e da fauna, garantindo equilíbrio entre as espécies benéficas e maléficas ao sistema produtivo, e a eliminação das queimadas.

Vários trabalhos têm demonstrado a maior presença de inimigos naturais das pragas e maior atividade microbiana nas áreas de SPD (Santos et al., 2005; Pereira et al., 2007). A biomassa microbiana é responsável pelo controle de funções essenciais no solo, como decomposição e acúmulo de matéria orgânica, ou por transformações que envolvem nutrientes minerais ou compostos no solo (Santos et al., 2005). O fato de o SPD apresentar normalmente maior biomassa microbiana pode proporcionar maior estocagem de nutrientes, possibilitando, também, melhor ciclagem destes ao longo do tempo, criando características mais favoráveis ao desenvolvimento das plantas. Essa maior estabilidade pode estar relacionada, também, com os fatores abióticos do solo, como o aumento da umidade, o incremento dos teores de matéria orgânica e a diminuição das temperaturas máximas do solo, favorecendo o crescimento das culturas (Salton \& Mielniczuk, 1995).

A utilização de herbicidas em sistema de integração entre milho e gramíneas forrageiras tem sido uma prática recomendada no controle da interferência exercida pela forrageira sobre a cultura, bem como no controle de plantas daninhas (Jakelaitis et al., 2005; Freitas et al., 2005). Entre os herbicidas utilizados, têm-se destacado o atrazine e algumas sulfonilureias, como o nicosulfuron. Na microbiota do solo, a atividade respiratória e o carbono da biomassa podem ser considerados, quando associados, indicadores da qualidade desse sistema, indicando desequilíbrios positivos ou negativos (Totola $\&$ Chaer, 2002).

Algumas técnicas têm-se mostrado eficientes na avaliação dos impactos dos cultivos agrícolas sobre o meio - a exemplo do emprego de indicadores microbiológicos para averiguação da qualidade do solo. Normalmente, pequenas alterações na qualidade do solo estão associadas com mudanças em suas propriedades microbiológicas, as quais apresentam alta sensibilidade a perturbações advindas do manejo (Pankhurst et al., 1997; Tótola et al., 2002).

Entre os indicadores microbiológicos de qualidade do solo, destacam-se a taxa respiratória (TR), a biomassa microbiana $(\mathrm{BM})$ e o quociente metabólico $\left(q \mathrm{CO}_{2}\right)$. A TR do solo é a medida da produção de $\mathrm{CO}_{2}$ resultante da atividade metabólica dos macro e microrganismos (Doran \& Parkin, 1994). A atividade desses organismos no solo é considerada um atributo positivo para a qualidade do solo e é usada como indicador por ser mais genérica e englobar a atividade de comunidades e consórcios de microrganismos presentes, apresentando melhor reprodutibilidade (Moorman, 1994; Schinner et al., 1996). Altas TRs do solo podem indicar distúrbio ecológico (exemplo, aplicação de agrotóxicos) ou alto nivel de produtividade do ecossistema solo (Islam \& Weil, 1998). A aplicação de agrotóxicos interfere positiva ou negativamente na atividade dos organismos do solo, propiciando a metabolização desses produtos pelos organismos e a capacidade de os agrotóxicos intoxicarem a biota do solo, respectivamente (Santos et al., 2005; Tuffi Santos et al., 2005; Vivian et al., 2006).

A biomassa microbiana do solo é considerada a parte viva da matéria orgânica do solo e inclui bactérias, actinomicetos, fungos, protozoários e algas. Em geral, as estimativas de biomassa são mais abrangentes, pois levam em consideração as populações microbianas cultiváveis e não cultiváveis (Lin \& Brookes, 1999). A BM está diretamente envolvida na degradação da matéria orgânica, na transformação e disponibilidade dos nutrientes e na degradação de agrotóxicos no solo (Angers et al., 1993; Moormam, 1994). A degradação de agrotóxicos pela BM do solo é amplamente relatada na literatura; no entanto, parte da BM (organismos não adaptados) pode ser afetada pelos agrotóxicos. Desse ponto de vista, ela destacase como importante indicador de qualidade do solo.

O quociente metabólico $\left(q \mathrm{CO}_{2}\right)$ consiste na taxa respiratória por unidade de $\mathrm{BM}$ do solo. Maiores valores de $\mathrm{qCO}_{2}$ sugerem condições 
desfavoráveis aos organismos do solo, e menores valores indicam maior eficiência da $\mathrm{BM}$ na utilização dos recursos do ecossistema, ou seja, menos carbono (C) é perdido como $\mathrm{CO}_{2}$ e maior proporção de $\mathrm{C}$ é incorporada nas células microbianas (Sakamoto \& Obo, 1994). $\mathrm{O} \mathrm{qCO}_{2}$ pode ser considerado o indicador mais adequado para avaliar o efeito das condições de estresse sobre a atividade da BM do solo (Anderson \& Domsch, 1993).

Dessa forma, objetivou-se com esta pesquisa avaliar o efeito do atrazine em mistura ao nicosulfuron sobre a atividade microbiológica do solo em sistema de cultivo convencional ou semeadura direta.

\section{MATERIAL E MÉTODOS}

O experimento foi conduzido em campo, na estação experimental pertencente à Universidade do Vale do Rio Doce (UNIVALE), em Governador Valadares (a $170 \mathrm{~m}$ de altitude, $18^{\circ} 34^{\prime} 30^{\prime \prime}$ de latitude sul e 41॰54'52" de longitude oeste). O estudo foi realizado em área de integração milho com braquiária. O plantio foi realizado em sulcos feitos após o preparo do solo, pelo sistema convencional de cultivo e sistema de plantio direto. A adubação foi realizada no momento do plantio, com base nos resultados da análise do solo e nas recomendações para a cultura (CFSEMG, 1999). A unidade experimental foi de $35 \mathrm{~m}^{2}$, constituída por cinco fileiras de milho de $7 \mathrm{~m}$ de comprimento e/ou 10 fileiras da braquiária.

Procedeu-se à análise química dos solos das áreas através da coleta de amostras simples da área avaliada, formando assim uma amostra composta, as quais foram encaminhadas ao laboratório de solos da UNIVALE para análise (Tabela 1). O plantio foi realizado em 10/5/2007, e a irrigação, conforme as necessidades da cultura.

O delineamento experimental utilizado foi de blocos casualizados em esquema fatorial $2 \times 4$, com quatro repetições. O fator A é representado pelo sistema de cultivo (sistema convencional e plantio direto), e o fator $\mathrm{B}$, pelos tratamentos (aplicações dos herbicidas atrazine + nicosulfuron em duas dosagens:

Tabela 1 - Composição química e textural da camada de 0-20 cm de profundidade do solo franco-arenoso, proveniente dos sistemas de plantio convencional e plantio direto utilizados no experimento. Governador Valadares-MG, 2007

\begin{tabular}{|c|c|c|c|c|c|c|c|c|c|c|}
\hline \multicolumn{11}{|c|}{ Sistema de plantio convencional } \\
\hline \multicolumn{11}{|c|}{ Fração mineral (\%) } \\
\hline \multicolumn{2}{|c|}{ Areia fina } & \multicolumn{2}{|r|}{ Areia grossa } & & \multicolumn{2}{|c|}{ Silte } & \multicolumn{2}{|l|}{ Argila } & \multicolumn{2}{|c|}{ Textura } \\
\hline \multicolumn{2}{|c|}{16,87} & \multicolumn{2}{|r|}{51,25} & & \multicolumn{2}{|c|}{22,09} & \multicolumn{2}{|l|}{9,8} & \multicolumn{2}{|c|}{ Franco arenosa } \\
\hline \multicolumn{11}{|c|}{ Análise química } \\
\hline $\mathrm{pH}$ & $\mathrm{P}$ & $\mathrm{K}+$ & $\mathrm{H}+\mathrm{Al}$ & $\mathrm{Al}^{3+}$ & $\mathrm{Ca}^{2+}$ & $\mathrm{Mg}^{2+}$ & $\mathrm{CTCt}$ & $\mathrm{V}$ & $\mathrm{m}$ & MO \\
\hline$\left(\mathrm{H}_{2} \mathrm{O}\right)$ & \multicolumn{2}{|c|}{$\left(\mathrm{mg} \mathrm{dm}^{-3}\right)$} & \multicolumn{5}{|c|}{$\left(\mathrm{cmol}_{\mathrm{c}} \mathrm{dm}^{-3}\right)$} & \multicolumn{2}{|c|}{$(\%)$} & $\left(\mathrm{dag} \mathrm{kg}^{-1}\right)$ \\
\hline 6,00 & 81,00 & 444,20 & 2,30 & 0,12 & 1,68 & 1,12 & 6,24 & 63,14 & 2,96 & 2,11 \\
\hline \multicolumn{11}{|c|}{ Sistema de plantio direto } \\
\hline \multicolumn{11}{|c|}{ Fração mineral (\%) } \\
\hline \multicolumn{2}{|c|}{ Areia fina } & \multicolumn{3}{|c|}{ Areia grossa } & \multicolumn{2}{|l|}{ Silte } & \multicolumn{2}{|l|}{ Argila } & \multicolumn{2}{|c|}{ Textura } \\
\hline \multicolumn{2}{|r|}{14,68} & \multicolumn{2}{|r|}{48,72} & & \multicolumn{2}{|l|}{12,50} & \multicolumn{2}{|l|}{24,10} & \multicolumn{2}{|c|}{ Franco-arenosa } \\
\hline \multicolumn{11}{|c|}{ Análise química } \\
\hline $\mathrm{pH}$ & $P$ & $\mathrm{~K}+$ & $\mathrm{H}+\mathrm{Al}$ & $\mathrm{Al}^{3+}$ & $\mathrm{Ca}^{2+}$ & $\mathrm{Mg}^{2+}$ & $\mathrm{CTCt}$ & $\mathrm{V}$ & $\mathrm{m}$ & $\mathrm{MO}$ \\
\hline $\mathrm{H}_{2} \mathrm{O}$ & \multicolumn{2}{|c|}{$\left(\mathrm{mg} \mathrm{dm}^{-3}\right)$} & \multicolumn{5}{|c|}{$\left(\mathrm{cmol}_{\mathrm{c}} \mathrm{dm}^{-3}\right)$} & \multicolumn{2}{|c|}{$(\%)$} & $\left(\right.$ dag kg $\left.^{-1}\right)$ \\
\hline 5,9 & 7,30 & 296,55 & 2,30 & 0,18 & 2,02 & 1,12 & 6,20 & 62,90 & 4,41 & 2,35 \\
\hline
\end{tabular}

Análises realizadas no Laboratório de Análises Físicas e Químicas de Solo do Núcleo de Pesquisa em Agronomia da Universidade Vale do Rio Doce. 
$1.500+10$ e $1.500+30 \mathrm{~g} \mathrm{ha}^{-1}$; e duas testemunhas: uma não capinada e capinada sem cultivo).

Os herbicidas foram aplicados em pósemergência do milho, quando este apresentava cinco folhas, aos 25 dias após o plantio, com pulverizador costal de precisão pressurizado a $\mathrm{CO}_{2}$, munido com barra de $2 \mathrm{~m}-$ acoplados a esta quatro pontas de pulverização da série TT 110.02 , espaçadas de 0,5 m e calibrado para aplicar o equivalente a $150 \mathrm{~L} \mathrm{ha}^{-1}$ de calda. No momento da aplicação a temperatura era de $23^{\circ} \mathrm{C}$, e a velocidade do vento, de $5 \mathrm{~km} \mathrm{~h}^{-1}$.

Por ocasião do florescimento do milho, foram retiradas amostras do solo em cada unidade experimental, na profundidade de 0-10 cm. Para determinação da taxa de desprendimento de $\mathrm{CO}_{2}$, as amostras de solo foram deixadas em temperatura ambiente, sendo, em seguida, peneiradas em malha de $4 \mathrm{~mm}$.

De cada amostra, $100 \mathrm{~g}$ tiveram sua umidade ajustada para $60 \%$ da capacidade de campo, sendo colocados em potes de plástico de capacidade de $2 \mathrm{~L}$, com tampa. Os potes foram acrescidos de um copo plástico $(50 \mathrm{~mL})$ contendo $20 \mathrm{~mL}$ de hidróxido de sódio $(\mathrm{NaOH})$ $1,0 \mathrm{~mol} \mathrm{~L}{ }^{-1}$, sendo a tampa posteriormente fechada, evitando a troca de ar entre o pote e o ambiente. Por meio da titulação do $\mathrm{NaOH}$, determinou-se o $\mathrm{CO}_{2}$ pelo método descrito por Stotzky (1965). Na determinação da biomassa microbiana foi empregado o método descrito por Vance et al. (1987), utilizando-se, em lugar do clorofórmio (fumigação), forno de microondas (irradiação) (Islam \& Weil, 1998). Estimou-se também o quociente metabólico $\left(q \mathrm{CO}_{2}\right)$, por meio da relação entre o $\mathrm{CO}_{2}$ acumulado ( $\mu \mathrm{g})$ e o $\mathrm{C}$ total da biomassa microbiana $\left(\mu \mathrm{g} \mathrm{g}^{-1}\right)$. Esse coeficiente mede a instabilidade do comportamento microbiano em função da avaliação da eficiência de utilização do carbono; maiores valores de $q \mathrm{CO}_{2}$ podem significar menor reutilização de carbono e, consequentemente, maior perda para atmosfera.

Os dados coletados foram submetidos à análise estatística pelo programa SAEG (Ribeiro Júnior, 2001), sendo as médias significativas comparadas pelo teste de Tukey a $5 \%$ de probabilidade.

\section{RESULTADOS E DISCUSSÃO}

Entre os sistemas de cultivo, a taxa de evolução do $\mathrm{CO}_{2}$ do solo foi menor somente nas parcelas não capinadas. Contudo, a comparação entre os tratamentos com e sem aplicação dos herbicidas evidenciou a menor taxa de evolução de $\mathrm{CO}_{2}$ nas amostras de solo proveniente das áreas sem cultivo, sendo mais acentuada no sistema de plantio direto (Tabela 2). Segundo Santos et al. (2007), a taxa de desprendimento de $\mathrm{CO}_{2}$ não pode ser avaliada como única característica da atividade microbiana do solo. Alterações do total de $\mathrm{CO}_{2}$ desprendido de amostras de solo podem ser produzidas em função de diferentes manejos, ficando, dessa forma, comprometidas avaliações isoladas dessa característica. Essa "respiração do solo" consiste num indicador sensivel da decomposição de resíduos, do giro metabólico do carbono orgânico e de distúrbios no ecossistema solo (Paul et al., 1999).

Comparando os sistemas convencional e plantio direto, constatou-se que em todos os casos, exceto para o solo sem cultivo, o CBM foi superior no sistema de plantio direto. Nas parcelas em que a mistura atrazine + nicosulfuron $\left(1.500+10 \mathrm{~g} \mathrm{ha}^{-1}\right)$ foi aplicada, $\mathrm{o}$ CBN foi de $125,05 \mu \mathrm{g} \mathrm{g} \mathrm{solo}{ }^{-1}$ nos solos onde o milho foi cultivado em sistema convencional e de $365,98 \mu \mathrm{g} \mathrm{g} \mathrm{solo}^{-1}$ no sistema de plantio direto; esses valores foram mais discrepantes

Tabela 2 - Valores para a taxa de desprendimento de $\mathrm{CO}_{2}$ em amostras de solo coletadas em áreas sob efeito de herbicidas para controle de plantas daninhas e manejo da braquiária em consórcio com milho (integração lavoura-pecuária). Governador Valadares-MG, 2007

\begin{tabular}{|c|c|c|}
\hline \multirow[b]{2}{*}{ Tratamento } & \multicolumn{2}{|c|}{$\mathrm{CO}_{2}$ diário $\left(\mu \mathrm{g} \mathrm{g} \mathrm{solo}^{-1}\right)$} \\
\hline & $\begin{array}{c}\text { Plantio } \\
\text { convencional }\end{array}$ & Plantio direto \\
\hline Solo sem cultivo $^{\underline{1}}$ & $110,88 \mathrm{Aa}$ & $102,56 \mathrm{Ab}$ \\
\hline Herbicidas $(1.500+10)^{\underline{2}}$ & $172,48 \mathrm{Aa}$ & $196,67 \mathrm{Aa}$ \\
\hline Herbicidas $(1.500+30)^{2 /}$ & $172,49 \mathrm{Aa}$ & $194,84 \mathrm{Aa}$ \\
\hline Sem capina ${ }^{3 /}$ & $161,56 \mathrm{Ba}$ & $200,23 \mathrm{Aa}$ \\
\hline $\mathrm{CV}(\%)$ & \multicolumn{2}{|c|}{11,69} \\
\hline
\end{tabular}

${ }^{1 /}$ Mantido capinado por todo o período. ${ }^{2 /}$ Doses $\left(\mathrm{g} \mathrm{ha}^{-1}\right)$ dos herbicidas (atrazine + nicosulfuron). ${ }^{3 /}$ Solo não capinado (testemunha sem herbicida). Para cada característica, médias seguidas por letras iguais, maiúsculas na linha e minúsculas na coluna, não diferem entre si, respectivamente, pelo teste $\mathrm{F}$ e de Tukey a $5 \%$ de probabilidade. 
entre os solos tratados com a mistura na dosagem de $1.500+30 \mathrm{~g} \mathrm{ha}^{-1}(412,12 \mathrm{e}$ $57,31 \mu \mathrm{g} \mathrm{g}$ solo $^{-1}$ nas parcelas cultivadas no sistema plantio direto e convencional, respectivamente) (Tabela 3). Apesar da elevada adsorção de herbicidas no solo, tem sido relatada a ação tóxica desses produtos sobre diversos grupos de organismos, principalmente do solo (Amarante Júnior et al., 2002; Santos et al., 2005, 2007). De acordo com Cattelan \& Vidor (1990), a atividade metabólica e o CBM são influenciados, entre outros fatores, pela temperatura, umidade, aeração e disponibilidade de substrato no solo, considerando que no solo cultivado no sistema de plantio direto esse substrato se encontra disponivel.

$\mathrm{Na}$ testemunha sem capina, observou-se também diferença entre os sistemas de cultivo: foi constatado que o CBM no plantio direto foi superior. Ao se compararem os tratamentos dentro do cultivo convencional, observou-se que nas parcelas onde a mistura atrazine + nicosulfuron foi aplicada na maior dose e nas áreas sem cultivo o CBM foi inferior, quando comparado ao das parcelas que receberam a menor dose da mistura herbicida e testemunha capinada (Tabela 3).

Os valores de biomassa microbiana do solo encontrados neste trabalho concordam com os obtidos por Santos et al. (2005) em solo cultivado com feijão e por Vivian et al. (2006) em solo cultivado com cana-de-açúcar; ambos os autores estimaram a biomassa microbiana pelo método utilizado neste trabalho. De modo geral, o CBM foi maior no sistema de plantio direto, independentemente da dose da mistura herbicida usada. A aplicação de herbicidas pode alterar a CBM do solo, porém esta apresenta resposta variável e depende do herbicida aplicado, do tipo de solo, da espécie de planta e da microbiota e suas interações. A interação herbicida-solo-microrganismo é demonstrada em alguns trabalhos - onde, por exemplo, o atrazine não provocou alterações na CBM de solo arenoso (Ghani et al., 1996) e, por outro lado, favoreceu o aumento da CBM em solo argiloso e com alto teor de matéria orgânica (Moreno et al., 2007). Em ambos os trabalhos não houve o cultivo de plantas.

Os herbicidas podem influenciar positiva ou negativamente a microbiota do solo e alterar duas ou mais características biológicas do
Tabela 3 - Valores para o carbono da biomassa microbiana (CBM) em amostras de solo coletadas em áreas sob efeito de herbicidas para controle de plantas daninhas e manejo da braquiária em consórcio com milho (integração lavourpecuária). Governador Valadares-MG, 2007

\begin{tabular}{|c|c|c|}
\hline \multirow[b]{2}{*}{ Tratamento } & \multicolumn{2}{|c|}{$\mathrm{CBM}\left(\mu \mathrm{g} \mathrm{g} \mathrm{\text {solo } ^ { - 1 } )}\right.$} \\
\hline & $\begin{array}{c}\text { Plantio } \\
\text { convencional }\end{array}$ & Plantio direto \\
\hline Solo sem cultivo ${ }^{1 /}$ & $44,39 \mathrm{Ab}$ & $58,58 \mathrm{Ac}$ \\
\hline Herbicidas $(1.500+10)^{2 /}$ & $125,05 \mathrm{Ba}$ & $365,98 \mathrm{Ab}$ \\
\hline Herbicidas $(1.500+30)^{\frac{2}{2}}$ & $57,31 \mathrm{Bb}$ & $412,12 \mathrm{Ab}$ \\
\hline Sem capina ${ }^{3 /}$ & $114,63 \mathrm{Ba}$ & $506,67 \mathrm{Aa}$ \\
\hline $\mathrm{CV}(\%)$ & \multicolumn{2}{|c|}{14,99} \\
\hline
\end{tabular}

1/ Mantido capinado por todo o período. ${ }^{2 /}$ Doses $\left(\mathrm{g} \mathrm{ha}^{-1}\right)$ dos herbicidas (atrazine + nicosulfuron). ${ }^{3 /}$ Solo não capinado (testemunha sem herbicida). Para cada característica, médias seguidas por letras iguais, maiúsculas na linha e minúsculas na coluna, não diferem entre si, respectivamente, pelo teste $\mathrm{F}$ e de Tukey a $5 \%$ de probabilidade.

solo de modo divergente - por exemplo, redução da biomassa microbiana e acréscimo da taxa respiratória - ou de modo convergente - nesse caso, redução ou acréscimo de ambas. Essas condições podem dificultar a interpretação correta dos efeitos de estresse sobre a biota do solo. Assim, para estimativas mais representativas desses efeitos, pode-se obter o $q \mathrm{CO}_{2}$, que relaciona a taxa respiratória com a biomassa microbiana. O quociente metabólico $\left(q \mathrm{CO}_{2}\right)$ foi proposto por Anderson \& Domsch (1985), estando diretamente relacionado ao fato de que, à medida que a biomassa de microrganismos do solo se torna mais eficiente na utilização dos recursos do ecossistema, menor quantidade de carbono se perde pela respiração e maior proporção desse elemento é incorporada aos tecidos microbianos. Nesse sentido, os maiores valores significam menor estabilidade dos microrganismos do solo ou menor grau de distúrbio.

Ao comparar os sistemas convencional e plantio direto, constatou-se que o $q \mathrm{CO}_{2}$ foi menor em solo cultivado no sistema de plantio direto para todos os tratamentos aplicados, evidenciando assim a maior estabilidade deste sistema de cultivo, o que se deve provavelmente à presença da camada de palha protegendo o solo no plantio direto. Dentro do sistema convencional as parcelas tratadas com a mistura atrazine+nicosulfuron (1.500 
$+30 \mathrm{~g} \mathrm{ha}^{-1}$ ) e o solo sem cultivo apresentaram maior $q \mathrm{CO}_{2}\left(3,01\right.$ e 2,50 $\mathrm{CO}_{2} / \mathrm{CBM}$, respectivamente), comparado aos tratamentos que receberam a mistura atrazine+nicosulfuron $\left(1.500+10 \mathrm{~g} \mathrm{ha}^{-1}\right)$ e testemunha sem capina (1,38 e 1,41 $\mathrm{CO}_{2} / \mathrm{CBM}$, respectivamente). No sistema de plantio direto, o tratamento sem cultivo mostrou-se o menos estável em relação aos demais, apresentando maior $q \mathrm{CO}_{2}$ (1,75 $\mathrm{CO}_{2}$ / CBM) (Tabela 4). Reis et al. (2008), trabalhando com efeito de herbicidas em solos cultivados com cana-de-açúcar, concluíram que o ametryn isolado e em mistura com trifloxysulfuron-sodium propiciou condições estressantes para a microbiota, sendo estas representadas pelos maiores valores de quociente metabólico do solo $\left(q \mathrm{CO}_{2}\right)$. Nesse mesmo trabalho, esses autores concluíram também que o 2,4-D e o trifloxysulfuron-sodium foram os herbicidas menos prejudiciais à microbiota do solo.

Em estudo de Santos et al. (2005) com herbicidas e sistemas de cultivo, foi observado que o $q \mathrm{CO}_{2}$ de solos preparados com aração e gradagem foi superior ao observado em semeadura direta, evidenciando menor condição de distúrbio neste último; que, da mesma forma, a aplicação de herbicidas promove aumento no $\mathrm{qCO}_{2}$ em solo sob preparo convencional; e que o efeito negativo de herbicidas sobre a microbiota do solo é reduzido em semeadura direta.

Tabela 4 - Valores para quociente metabólico $\left(q \mathrm{CO}_{2}\right)$ em amostras de solo coletadas em áreas sob efeito de herbicidas para controle de plantas daninhas e manejo da braquiária em consórcio com milho (integração lavoura-pecuária). Governador Valadares, 2007

\begin{tabular}{|c|c|c|}
\hline \multirow[b]{2}{*}{ Tratamento } & \multicolumn{2}{|c|}{$q \mathrm{CO}_{2}\left(\mathrm{CO}_{2} / \mathrm{CBM}\right)$} \\
\hline & $\begin{array}{c}\text { Plantio } \\
\text { convencional }\end{array}$ & Plantio direto \\
\hline Solo sem cultivo $^{1 /}$ & $2,50 \mathrm{Aa}$ & $1,75 \mathrm{Ba}$ \\
\hline Herbicidas $(1.500+10)^{\frac{2}{}}$ & $1,38 \mathrm{Ab}$ & $0,54 \mathrm{Bb}$ \\
\hline Herbicidas $(1.500+30)^{\frac{2}{}}$ & $3,01 \mathrm{Aa}$ & $0,47 \mathrm{Bb}$ \\
\hline Sem capina ${ }^{3 /}$ & $1,41 \mathrm{Ab}$ & $0,39 \mathrm{Bb}$ \\
\hline $\mathrm{CV}(\%)$ & \multicolumn{2}{|c|}{18,55} \\
\hline
\end{tabular}

1/ Mantido capinado por todo o período. ${ }^{2 /}$ Doses $\left(\mathrm{g} \mathrm{ha}^{-1}\right)$ dos herbicidas (atrazine + nicosulfuron). ํ/ Solo não capinado (testemunha sem herbicida). Para cada característica, médias seguidas por letras iguais, maiúsculas na linha e minúsculas na coluna, não diferem entre si, respectivamente, pelo teste $\mathrm{F}$ e de Tukey a $5 \%$ de probabilidade.
Pode-se concluir que os indicadores microbiológicos avaliados foram sensiveis aos tratamentos propostos, indicando menor distúrbio ao solo quando em sistema de semeadura direta. Entretanto, na integração lavoura-pecuária em sistema convencional de cultivo, o efeito negativo da aplicação dos herbicidas é aumentado com o incremento na dose do nicosulfuron.

\section{AGRADECIMENTOS} dido.

Ao CNPq e à FAPEMIG, pelo apoio conce-

\section{LITERATURA CITADA}

AMARANTE JUNIOR, O. P. et al. Glyphosate: properties, toxicity, use and legislation. Química Nova, v. 25, n. 4, p. 589-593, 2002.

ANDERSON, J. P.; DOMSCH, K. H. The metabolic quotient for $\mathrm{CO}_{2}\left(q \mathrm{CO}_{2}\right)$ as a specific activity parameter to asses the effects of environmental conditions, such as $\mathrm{pH}$, on the microbial biomass of forest soils. Soil Biol. Biochem., v. 25, n. 3, p. 393-395, 1993.

ANDERSON, T. H.; DOMSCH, K. H. Determination of ecophysiological maintenance carbon requirements of soil microorganisms in a dormant state. Biol. Fert. Soils, v. 1, n. 1, p. $81-89,1985$

ANGERS, D. A. et al. Tillage-induced differences in organic matter of particle-size fractions and microbial biomass. Soil Sci Am. J., v. 57, n. 2, p. 512-516, 1993.

CATTELAN, A. J.; VIDOR, C. Flutuações na biomassa, atividade e população microbiana do solo, em função de variações ambientais. R. Bras. Ci. Solo, v. 14, n. 1, p. 133-142, 1990.

COMISSÃO DE FERTILIDADE DO SOLO DO ESTADO DE MINAS GERAIS - CFSEMG. Recomendações para o uso de corretivos e fertilizantes em Minas Gerais - $5^{\text {a }}$ aproximação. Viçosa, MG: Universidade Federal de Viçosa, 1999. 359 p.

DORAN, J. W.; PARKIN, T. B. Defining and assessing soil quality. In: DORAN, J. M. (Eds.). Defining soil quality for a sustainable environment. Madison: Soil Science Society of America, 1994. p. 3-21. (Special Publication, 35).

FEDERAÇÃO BRASILEIRA DE PLANTIO DIRETO NA PALHA - FEBRAPDP (2008) Ponta Grossa, Boletim Informativo. Ano 9, n. 33, 08 p. 
FREITAS, F. C. L. et al. Formação de pastagem via consórcio de Brachiaria brizantha com milho para silagem no sistema de plantio direto. Planta Daninha, v. 23, n. 1, p. 49-58, 2005.

GHANI, D. A. et al. Interactions between ${ }^{14} \mathrm{C}$-labelled atrazine and the soil microbial biomass in relation to herbicide degradation. Biol. Fert. Soils, v. 21, n. 1, p. 17-22, 1996.

ISLAM, K. R.; WEIL, R. R. Microwave irradiation of soil four routine measurement of microbial biomass carbon. Biol. Fert. Soils, v. 27, n. 4, p. 408-416, 1998.

JAKELAITIS, A. et al. Influência de herbicidas e de sistemas de semeadura de Brachiaria brizantha consorciada com milho. Planta Daninha, v. 23, n. 1, p. 59-68, 2005.

LIN, Q.; BROOKES, P. C. Comparison of substrate induced respiration, selective inhibition and biovolume of microbial biomass and its community structure in unamended, ryegrassamended, fumigated and pesticide-treated soils. Soil Biol.

Biochem., v. 31, n. 3, p. 1999-2114, 1999.

MOORMAN, T. B. Pesticide degradation by soil microrganisms: environmental, ecological and management effects. In: HATFIELD, J. L.; STEWART, B. A. (Eds.). Soil biology. Effects on soil quality. Boca Raton: CRC Press, 1994. p. 121-169.

MORENO, J. L. et al. Effects of atrazine on microbial activity in semiarid soil. Appl. Soil Ecol., v. 35, n. 1, p. 120-127, 2007.

PANKHURST, C. E.; DOUBE, B. M.; GUPTA, V. V. S. R. (Eds.). Biological indicators of soil health. Boca Raton: CRC Press, 1997. 268 p.

PAUL, E. A. et al. Evolution of $\mathrm{CO} 2$ and soil carbon dynamics in biologically managed, rowcrop agroecosystems. Appl. Soil Ecol., v. 11, n. 1, p. 53-65, 1999.

PEREIRA, J. L. et al. Growth inhibitory effects of nutgrass (Cyperus rotundus) on rice (Oryza sativa) eedlings. J. Chem. Ecol., v. 26, n. 9, p. 2221-2231, 2007.

REIS, M. R. et al. Atividade microbiana em solo cultivado com cana-de-açúcar após aplicação de herbicidas. Planta Daninha, v. 26, n. 3, p. 323-331, 2008.

RIBEIRO JÚNIOR, J. I. Análises estatísticas no SAEG Viçosa, MG: Universidade Federal de Viçosa, 2001. 301 p.
SAKAMOTO, K.; OBO, Y. Effects of fungal to bacterial ratio on the relationship between $\mathrm{CO}_{2}$ evolution and total soil microbial biomass. Biol. Fert. Soils, v. 17, n. 1, p. 39-44, 1994.

SALTON, J. C.; MIELNICZUK, J. Relações entre sistemas de preparo, temperatura e umidade em um Podzólico Vermelho-Escuro de Eldorado do Sul (RS). R. Bras. Ci. Solo, v. 19 , n. 2 , p. $313-319,1995$.

SANTOS, J. B. et al. Atividade microbiana do solo após aplicação de herbicidas em sistemas de plantio direto e convencional. Planta Daninha, v. 23, n. 4, p. 683-691, 2005

SANTOS, J. B. et al. Avaliação de formulações de glyphosate sobre soja Roundup Ready. Planta Daninha, v. 25, n. 1, p. 165-171, 2007.

SCHINNER, F. et al. (Eds.). Indirect estimation of microbial biomass. In: SPARLING, G. P. et al. Methods in soil biology. Heidelberg: Springer-Verlag, 1996. p. 47-75.

STOTZKY, G. Microbial respiration. In: BLACK, C. A. Methods of soil analysis. Madison: Americam Society of Agronomy, 1965. v. 2. p. 1151-1572.

TÓTOLA, M. R.; CHAER, G. M. Microrganismos e processos microbiológicos como indicadores da qualidade dos solos. In: ALVAREZ V; V.H. et al. (Eds.). Tópicos em Ciência do Solo. Viçosa, MG: Sociedade Brasileira de Ciência do Solo, 2002. v. 2. p. 195-276.

TÓTOLA, M. R.; CHAER, G. M. Microrganismos e processos microbiológicos como indicadores da qualidade dos solos. In: ALVAREZ V. V. H. et al. (Eds.). Tópicos em Ciência do Solo. Viçosa, MG: Sociedade Brasileira de Ciência do Solo, 2002. v. 2. p. 195-276.

TUFFI SANTOS, L. D. et al. Exsudação radicular do glyphosate por Brachiaria decumbens e seus efeitos em plantas de eucalipto e na respiração microbiana do solo. Planta Daninha, v. 23, n. 1, p. 143-152, 2005.

VANCE, E. D.; BROOKES, P. C.; JENKINSON, D. S. An extraction method for measuring soil microbial biomass $\mathrm{C}$. Soil Biol. Biochem., v. 19, n. 4, p. 703-707, 1987.

VIVIAN, R. et al. Persistência de sulfentrazone em Latossolo Vermelho-Amarelo cultivado com cana-de-açúcar.

Planta Daninha, v. 24, n. 4, p. 741-750, 2006. 